\title{
940 TARGETING DKK1 TO REVERSE IMMUNOSUPPRESSIVE MACROPHAGES AND ENHANCE PD-1 BLOCKADE THERAPY IN GASTRIC CANCER
}

\footnotetext{
${ }^{1}$ Tao Shi*, ${ }^{1} Y i p e n g$ Zhang, 'Yue Wang, 'Yunfeng Pan, 'Hanbing Wang, ${ }^{1} Y u t i n g$ Luo, ${ }^{1}$ Kaijie Liang, ${ }^{2}$ Fangcen Liu, ${ }^{1}$ Qin Liu, ${ }^{1}$ Keying Che, ${ }^{3}$ Xuan Wang, ${ }^{1}$ Lixia Yu, ${ }^{1}$ Baorui Liu, ${ }^{1}$ Jia Wei. 'The Comprehensive Cancer Centre of Drum Tower Hospital, Medical School of Nanjing University and Clinical Cancer Institute of Nanjing University, Nanjing, China., Nanjing, China; ${ }^{2}$ Pathology Department, Affiliated Drum Tower Hospital to Medical School of Nanjing University, Nanjing, China, Nanjing, China; ${ }^{3}$ The Comprehensive Cancer Centre of Nanjing Drum Tower Hospital, Clinical College of Nanjing Medical University., Nanjing, China
}

Background Gastric cancer (GC) is a highly heterogeneous and immunosuppressive cancer type with poor prognosis. Current immunotherapies like immune checkpoint blockade (ICB) have very modest therapeutic effect in GC patients, reflecting urgent need for exploring new immunotherapeutic targets.

Methods IHC and mRNA analysis of 384 patients from Drum Tower Hospital Cohort and 1192 patients from other databases were performed to investigate Dickkopf-1 (DKK1) expression and local immune status. The MFC-challenged subcutaneous and abdominal dissemination GC models were established, and the impact of DKK1 blockade on gastric tumor immune microenvironment (TIME) and anti-tumor responses was explored by flow cytometry and RNA sequencing. In vivo immune cell-depletion GC models were constructed to further assess the function of DKK1 on different immune cell types. RAW264.7 and mouse bone marrow derived macrophages (BMDMs) were employed to analyze DKK1 modulation on macrophages in vitro by Cytometric Bead Array, flow cytometry and western bolt.

Results In present study, we found high DKK1 expression is associated with poor overall survival and worse immune status in GC patients. DKK1 blockade could improve gastric TIME, including increased accumulation and activation of CD8 $+\mathrm{T}$ cells and NK cells, and trigger an effective anti-tumor response both in subcutaneous and abdominal dissemination GC models. DKK1 directly induces macrophages towards an immunosuppressive phenotype, while the TIME improvement and tumor reduction depend on the reversion of immunosuppressive macrophages mediated by DKK1 blockade. Furthermore, combined inhibition of PD-1 and DKK1 could achieve superior anti-tumor effect on GC models.

Conclusions Thus, our work identifies a new role of DKK1 to induce immunosuppressive TIME through macrophage modulation, and reveals DKK1 to be a novel and promising immunotherapeutic target for GC.

Ethics Approval The collection and analysis of tumor tissue sections were approved by the Ethics Committee of Nanjing University Medical School Affiliated Drum Tower Hospital (2021-324-01). All animal experiments were approved by the Institutional Animal Care and Use Committee of Drum Tower Hospital (approval number: 2020AE01064).

http://dx.doi.org/10.1136/jitc-2021-SITC2021.940 